\title{
El profesorado de ciencias y matemáticas y la comunicación a través de las TIC
}

\author{
David MÉndez CocA \\ Centro Universitario Villanueva de Madrid \\ dmendez@villanueva.edu \\ Miriam MÉNDEZ CoCA \\ Centro Universitario Villanueva de Madrid \\ nmendez@villanueva.edu
}

\begin{abstract}
Resumen:
La integración de las tic en la educación avanza positivamente superando las barreras de la falta e inadecuada formación de los profesores de matemáticas y ciencias y la escasez de recursos en los centros. Los avances de la tecnología y su adecuada aplicación a la educación permiten una personalización del aprendizaje, un incremento de la motivación en los estudiantes y un enriquecimiento de las prácticas didácticas.
\end{abstract}

Palabras clave: Tic; recursos metodológicos; enseñanza con móviles.

The math and science teacher and the communication with the itc.

\begin{abstract}
:
The integration the ict in education progress successfully exceeding the barriers of the lack and unsuitable training of math and science teachers and the lack of means in schools. The advance of technology and the proper application of this technology to the education allow a personalized teaching and an improvement of the motivation in the students and enrichment of the didactic practice.
\end{abstract}

Key Words: ITC; didactic methods; teaching with smartphones.

\section{Referencia normalizada:}

Méndez Coca, D y Méndez Coca, M. (2014): El profesorado de ciencias y matemáticas y la comunicación a través de las TIC. Historia y Comunicación Social. Vol. 19. Núm. Especial Enero. Págs. 315-326.

Sumario: 1. Introducción 2. Actualidad tecnológica 3. Programas de instituciones educativas para la integración de la tecnología en educación 4. La tecnología y la formación del estudiante 5. Herramientas tecnológicas en el aula 5.1. Los ordenadores en el aula 5.2. Los dispositivos móviles en el aula 5.3. Las pizarras digitales 6 . Conclusiones

\section{Introducción}

En la actualidad el mundo de la tecnología está entrando a formar parte del mundo educativo generado diversas situaciones problemáticas a las que hay que dar respuesta. 
En la sociedad actual crece y se desarrolla con fuerza un nuevo espacio social, el espacio digital, como fruto de la convergencia de la tecnología y de una serie de factores sociales y culturales favorables a los avances tecnológicos. Resulta de interés la referencia a algunos aspectos que tienen mayor incidencia en el área de la educación: la tecnología actúa de soporte de almacenamiento de la información y el conocimiento haciéndolos accesibles y favoreciendo su comunicación y trasmisión.

En cuanto soportes de almacenamiento de información la tecnología es receptora de los conocimientos precedentes que el hombre ha elaborado y guardado sobre sí mismo, sobre la naturaleza y sobre la ciudad que «pueden ser transferidos al entorno electrónico» (Echevarría, 2000: 27) arrastrando los costes que ello exigiera.

La integración de la tecnología en el ámbito educativo ha presentado varias dificultades, pero ha sido positiva puesto que propicia entornos educativos que amplían considerablemente las posibilidades del sistema, no sólo de tipo organizativo, sino también de transmisión de conocimientos y desarrollo de competencias y actitudes. La clave está en transformar la información en conocimiento, y éste en educación y aprendizaje significativo (Méndez, 2012a).

\section{Actualidad tecnológica}

Las tecnologías que se utilizan en el mundo educativo hoy día son internet, proyector, pizarra digital, portátil, tablet y móvil principalmente si nos referimos al hardware. En cuanto a las opiniones que generan estas tecnologías de la información y comunicación, TIC, aplicadas a la educación existen diversos estudios, aquí destaco uno realizado desde 2001 hasta 2007 (Sigalés y Momino, 2009), con una selección de 700 escuelas e institutos, con un total de 17.000 cuestionarios y entrevistas, llegaron a las siguientes conclusiones:

- El 60\% de los profesores consideran que el tiempo de preparación de una clase con TIC es sustancialmente mayor que el necesario para una clase convencional.

- Los profesores de infantil y primaria manifiestan que estas tecnologías son de poca ayuda.

- El 31\% del profesorado afirma que el uso de las TIC mejora los resultados académicos, en cambio el resto defiende que no mejoran e incluso empeoran.

Como se puede observar las opiniones contrarias a aplicar las TIC existen, no obstante, a medida que transcurre el tiempo, aumenta el número de autores y profesores que se muestran dispuestos a la integración de las TIC en el sistema educativo, incluso señalan el recurso material de las nuevas tecnologías como un factor de la calidad educativa (Climent Giné, 2002). En España, los organismos estatales y autonómicos siguen estimulando el aumento de la ratio de ordenador por alumno: desde 
el 2002 hasta el 2010 ha pasado de 13,4 alumnos por ordenador a 5 (INE, 2011). Las ventajas que ofrecen los medios tecnológicos se pueden sintetizar en las siguientes (Aguiar y Cuesta, 2009):

- Influyen en la vida diaria del alumno y en la educación informal, coincidiendo con la educación formal con las ventajas que ello comporta.

- Estimulan la comunicación interpersonal y ofrecen múltiples posibilidades de aplicación.

- Desarrollan en el alumnado la capacidad investigadora.

A pesar de estas ventajas, las TIC, todavía no se emplean con gran asiduidad (Clares y Gil, 2008). Sin embargo, cada vez más los profesores coinciden en mayor número con los estudiantes en la utilidad de las TIC para el desarrollo de sus habilidades y para la comprensión de los contenidos educativos (Edmunds et al., 2012). No obstante, para un aprendizaje satisfactorio de los contenidos, el proceso de implantación de las TIC exige un diseño de planificación de actividades, realizado con minuciosidad y compartiendo experiencias con profesores de la especialidad (Vázquez, 2011; Méndez, 2012b). El uso de estos instrumentos en el proceso de la enseñanza facilita unas clases más participativas y activas por parte del alumnado (Aguaded-Gómez et al., 2010).

Es cierto que los profesores reconocen el valor de este recurso en la educación, experimentan dificultades a la hora de adoptar dicha tecnología. En consecuencia, solo una minoría de docentes las ha incorporado a la enseñanza. Entre las barreras que existen para el uso de las TIC, se menciona (Méndez, 2012c):

- La falta de conocimientos del profesorado

- Una escasa motivación y confianza en el uso de dichas tecnologías

- Una formación inadecuada,

- La ausencia o escasas calidad de infraestructura para las TIC y otras cuestiones relacionadas con los sistemas educativos tradicionales.

El informe concluye que, para asegurar unas soluciones políticas realistas e integrales, es necesario identificar y comprender todos los factores que impiden que el profesorado las integre de manera plena en su práctica docente (Eurydice, 2011).

Por tanto, se observa que la investigación y las instituciones están trabajando para realizar una integración de las tecnologías, todo esto tiene como fin que los estudiantes tengan un mayor aprendizaje.

Se perciben dos perspectivas de formación complementarias: una es la formación de los estudiantes en los avances de la tecnología, la formación mas técnica, y otra es la formación de los estudiantes en valores, la formación más humanista, con el fin de facilitar la creación de una sociedad más democrática, solidaria y respetuosa con el hombre y con la naturaleza. Ante estas perspectivas de cambio en la educación, la implicación del profesorado y de las instituciones educativas es imprescindible, 
puesto que el profesor es quien ha de responder a estas perspectivas en el aula y son las instituciones las llamadas a organizar esta formación de la forma más eficiente.

\section{Programas de instituciones educativas para la integración de la tecnología en educación}

La formación de los alumnos en los avances tecnológicos exigen un requisito previo: el profesor ha de tomar conciencia del desarrollo tecnológico y de los avances de la nanociencia para que los nuevos materiales se incorporen a la educación formal de los estudiantes en edad escolar. La situación hace necesario que los profesores sean preparados para impartir estos conceptos y sus implicaciones (Sweeney, Seal y Vaidyanathan, 2003). La formación del profesor tiene consecuencias sociales de las que deben ser conscientes las instituciones educativas. Uno de los movimientos que se está impulsando desde las organizaciones gubernamentales en USA es el Proyecto 2061: "Ciencia para todos los americanos anima a los profesores de ciencia a introducir asuntos sociales y tecnológicos dentro del tejido del currículo de las ciencias" (Pedersen y Totten, 2001: 376).

En Europa se está impulsando contextualizar "la enseñanza de la ciencia, también denominado ciencia-tecnología-sociedad. Este enfoque enfatiza los aspectos filosóficos, históricos o sociales de la ciencia y la tecnología, a la vez que conecta con la comprensión de las experiencias diarias de los estudiantes." Se considera que el procedimiento va "a aumentar la motivación y el conocimiento científico" (Eurydice, 2011: 64-65). Este enfoque proporciona una educación científica más coherente y exhibe un marco de gran potencial para promover el aprendizaje de los estudiantes (Keller y Pearson, 2012).

En Estados Unidos, “este enfoque surge como respuesta a la crisis que comenzó a aflorar a comienzos de los años sesenta en la relación que mantenía la sociedad con la ciencia y la tecnología" (Membiela, 1997: 51). En este enfoque se pueden distinguir hasta seis formas diferentes de introducir las relaciones de ciencia, tecnología y sociedad: aplicación y diseño, histórico, argumentación lógica, centrado en los valores, sociocultural y relacionado con la justicia social y con el ecologismo (Pedretti y Nazir, 2011).

También hay que tomar en consideración que la tecnología aparece en estrecha relación con la economía, la ciencia y la sociedad, en especial en los medios de comunicación (Colas, 2002). Por tanto, esta inclusión en el currículo sería aceptada por parte del alumnado de una forma natural. Se ha de añadir además que esta incidencia en las relaciones entre la ciencia, la tecnología y la sociedad puede llevar a "una enseñanza en la se proporciona un contexto apropiado para el aprendizaje basado en la investigación" (Havu-Nuutinen y Keinonen, 2010: 221). En consecuencia, la inclusión de las relaciones de la ciencia, tecnología y sociedad en el currículo conlle- 
varía un cambio didáctico, un cambio en la enseñanza para el que hay que preparar al profesorado.

\section{La tecnología y la formación del estudiante}

La sociedad del conocimiento exige que los estudiantes se capaciten en el manejo de las nuevas tecnologías, a fin de aplicarlas en el trabajo. La educación no puede lograr sus objetivos de espaldas a esta necesidad, más bien ha de asumir su papel en la innovación y en el cambio sociocultural, a fin de impartir una formación en sintonía con las exigencias de la sociedad globalizada. Desde la actual convergencia de cambios se exige que el estudiante ubicado en el espacio digital, donde el acceso a la información es sencillo y rápido, tenga el propósito de avanzar en la autonomía personal y en la capacidad de gestión del cambio, manteniendo la suficiente flexibilidad e interés para seguir aprendiendo a lo largo de toda la vida.

La innovación que provoca Internet y las demás tecnologías por las que se accede a la red afecta a la información, a los escenarios sociales, a los contenidos científicos, económicos y políticos sobre los que se teje la totalidad de la actividad humana. La comunicación e interacción entre los individuos se lleva a cabo mediante otras tecnologías y se ha de procurar que los alumnos sean capaces de adaptarse a las nuevas situaciones (Colás, 2002).

La educación tiene que asumir el doble reto de formar al estudiante como ciudadano activo y responsable y ocuparse asimismo de su preparación técnica. En ningún caso cumpliría su objetivo esencial si el sistema educativo renunciara a alguno de las dos exigencias. Esta razón hace atractivos los estudios interdisciplinarios porque "responden a una de las preguntas más usuales de los estudiantes: ¿por qué? Las materias tradicionales a menudo llevan a los estudiantes a preguntarse sobre la relevancia de sus estudios; notan que los conocimientos de la clase y el aprendizaje del libro se aplican poco para el desarrollo de habilidades, el establecimiento de fundamentos y la comprensión de verdades". (Mei, 2009: 41-42)

Estos estudios pueden evitar el peligro de vivir bajo la lógica tecnológica, consiguen de los alumnos una mente más abierta al relacionarse diferentes saberes, además de asumir la responsabilidad ante la aplicación de estas herramientas. En la sociedad globalizada del conocimiento es más necesario que en otras épocas que los estudiantes ejerzan la habilidad de pensar y actuar como ciudadanos responsables en la sociedad tecnológica, porque en ésta las acciones y razonamientos están definidos en función de la lógica de la tecnología (Marino y Hayes, 2012). Muchos estudiantes no llegan a entender la vida sin móvil o sin internet, en cambio no encuentran placer en el intercambio de opiniones tan enriquecedor para el ser humano, en la contemplación de los fenómenos naturales, en reflexionar un acontecimiento, en la lectura de un libro de denso contenido. 
Para concluir, insistimos en que la formación del estudiante no debe contener sólo aspectos técnicos o científicos sino también un conjunto de principios que le permita valorar los problemas que genera el mal uso de la tecnología. Es cada vez más habitual el acoso entre compañeros en la escuela, en España el índice de acoso es del 23\% y es muy habitual que la mayor parte de las formas de acoso se practiquen mediante el mal uso de las nuevas tecnologías (Yasuda, 2010).

\section{Herramientas tecnológicas en el aula}

En el aula se hace uso de los ordenadores e internet principalmente, además se utilizan los dispositivos móviles: los tablets y los móviles.

\subsection{Los ordenadores en el aula}

Dentro de la tecnología, la más antigua utilizada es el ordenador, suele ser la que tiene mayor material accesible para la educación. De hecho se suele decir que ocupa un lugar especial (Méndez, 2014). Los ordenadores abren un amplio rango de posibilidades, puede aumentar la autonomía de los estudiantes siempre que se emplee el software adecuado, por ejemplo para conceptos de química existen el ComLab, CAI para química, KineticsLab y Concept Builder (Orlik, 2002), además también existen laboratorios virtuales que se han implementado, hoy día están sustituyendo a los laboratorios tradicionales, en cuanto al efecto que está causando en el aprendizaje de los estudiantes se ha visto muy positivo (Zacharia \& Olympiou, 2011; Donnelly et al., 2011). Otras experiencias que se han aplicado de forma positiva son la utilización de sistemas electrónicos de respuesta y la resolución de problemas (Scherer \& Tiemann, 2012; Donovan, 2008).

Otra gran ventaja de los ordenadores consiste en que facilitan el uso de todos los recursos de internet al aula, estos recursos también se han visto que ayudan a los estudiantes a la hora del aprendizaje que logran, especialmente las animaciones (Kortemeyer, 2006; Wilder \& Brinkerhoff, 2007). Incluso los ordenadores pueden "reducir la carga cognitiva permitiendo a los estudiantes dirigirse hacia procesos de orden más alto" (Josephsen \& Kristensen, 2006:276).

Otro efecto positivo de la integración de los ordenadores es el aumento de motivación e interés que es una ayuda importante de cara a la comprensión de los conceptos (Su, 2008; Martín-Villalba et al., 2012).

\subsection{Los dispositivos móviles en el aula}

Los estudiantes usan Smartphone, el uso de portátiles está muy extendido pero no tanto como el móvil. La portabilidad hace posible dispositivos móviles porque los estudiantes pueden acceder y estar conectados todo el tiempo (Melhuish \& Falloon, 2010). Además la familiaridad de los estudiantes con estos dispositivos puede ser una 
gran ayuda en el campo educativo. De hecho, los smartphones es un tipo de dispositivo que pueden cambiar el proceso de instrucción (Eisele-Dyrli, 2011).

En cuanto a los datos, Estados Unidos, por ejemplo, el 98\% de los estudiantes entre catorce y dieciocho años tienen su propio móvil y el 70\% tienen en propiedad un portátil, tablet o netbook (Project Tomorrow, 2010).

Las tecnologías conllevan un atractivo para los estudiantes que promueve una extra de motivación que puede ser muy interesante. En Estados Unidos, el 93\% de los padres les gusta la idea de libros de texto online, el $47 \%$ considera que los libros de texto digitales serían una buena inversión para los colegios con el fin de aumentar el rendimiento de los estudiantes (Project Tomorrow, 2010: 25).

Una de las grandes ventajas que tiene el profesor al utilizar esta tecnología es que no hace falta formación ad hoc, ni tampoco formar a los estudiantes. Además aunque al inicio parece que el profesor tiene que trabajar un tiempo extra, a la larga el ahorro de tiempo y el aumento de eficacia se hacen más patentes (Kolb, 2011).

Existen varias páginas web que pueden facilitar las tareas del profesor. Los estudiantes pueden acceder a ellas de forma sencilla y rápida con cualquier dispositivo móvil con conexión a internet. Dentro de estas webs destacan poll everywhere, Go Soapbox and socrative (Matthew, 2012).

Son los llamados smart student response systems, esto es, los sistemas inteligentes de respuesta para estudiantes. Sólo se necesita conexión a internet y un dispositivo por el cual se pueda conectar. El profesor diseña las actividades o problemas y el estudiante entra en la web por medio del número o contraseña que le da el profesor y responde. El profesor puede ver el archivo con las respuestas en un Excel inmediatamente.

Estos sistemas no deben evitar el diálogo ni el aprendizaje cooperativo, más bien al contrario, de hecho puede ser muy interesante de cara a mejorar la eficacia del aprendizaje cooperativo, juntando a los estudiantes que tengan diferentes respuestas ante una pregunta.

Las ventajas de los móviles son las siguientes (Attewell, 2005; Kolb, 2011; Duncan et al., 2012):

El profesor puede diseñar fácilmente las preguntas.

1. Los estudiantes pueden decidir con gran libertad las respuestas.

2. El profesor conoce los resultados enseguida.

3. Los resultados no son públicos.

4. El aprendizaje por móvil puede animar la autonomía y el aprendizaje cooperativo.

5. Quita formalidad a las experiencias de aprendizaje.

6. Ayuda a aumentar la autoestima. 
7. Hace que los estudiantes puedan estar más centrados más tiempo.

8. Ahorran dinero.

9. Ayuda a combatir la resistencia existente al uso de las TIC.

10. Los estudiantes los utilizan muy bien.

11. Son una herramienta flexible.

12. Pueden potenciar tanto el aprendizaje visual como auditivo.

13. Distraen menos que los portátiles.

Hoy día en la literatura de la investigación educativa, se han realizado experimentos con efecto positivo utilizando el móvil en el aula (Méndez y Slisko, 2013).

\subsection{Las pizarras digitales}

En diferentes lugares, cada vez en más colegios e institutos se han equipado de pizarras digitales interactivas, pdi. El fin de esta inversión es tener unas clases más participativas, con material digital e introducir las animaciones que se encuentran en el mundo de internet.

En cuanto al papel del profesorado, se realizan cursos de formación con escasa participación debido a los horarios exigentes que tienen en dicha profesión. En consecuencia, a esta herramienta tecnológica no se le está sacando todo el provecho que se pudiera.

Aún así, existen experiencias que se han realizado utilizando sólo el proyector y el resultado también ha sido muy positivo (Méndez, 2012b; Méndez, 2013).

\section{Conclusiones}

La integración del mundo tecnológico en el aula y el avance tan veloz de estas herramientas, junto con el desarrollo del material interactivo ha generado una serie de dimensiones a las que se les debía haber dado una respuesta, no obstante aún no se le ha dado porque el problema es mayor del que se preveía.

Se esperaba que el profesor estuviera al día de las herramientas tecnológicas y supiera utilizarlas en el aula, además también se le pedía que estuviera al día del material de internet. En cambio, se observa que el profesor no está suficientemente formado en la utilización de estas herramientas, existen algunos profesores que sí tienen dicha formación.

Debido a la presión de las instituciones educativas y de las familias de los estudiantes principalmente, los centros escolares y los profesores están integrando la tecnología como si fuera un fin, por tanto esta herramienta que debiera facilitar dos 
objetivos: fomentar el aprendizaje cooperativo y la educación personalizada, no está cumpliéndolos.

Por estas razones, se hace necesario una inversión en la formación del profesorado, no sólo en material para el aula sino en modos de utilización e integración en la clase del día a día.

Es importante que estas herramientas faciliten la educación personalizada, por tanto desde aquí se hace un llamamiento a las instituciones educativas para que inviertan en la creación de materiales curriculares en internet de libre acceso, de esta forma será más sencillo que el profesor atienda a cada ritmo de aprendizaje.

Otra cuestión que no es baladí es fomentar con las tecnologías la interacción entre los estudiantes, el aprendizaje cooperativo, mediante cualquier metodología de enseñanza (Méndez y García-Alonso, 2013). Con estas herramientas es más sencillo conocer las opiniones de los estudiantes y así, facilitar la formación de grupos en el aula con alumnos que opinen de diferente forma o que tengan un nivel de aprendizaje distinto.

Si la tecnología se introduce en el aula sin previa formación del profesorado, los alumnos tendrán una herramienta para distraerse en el aula y no sacar todo el provecho posible de las explicaciones del profesor, por tanto lograrán unos resultados peores de los conseguidos hasta ahora. No obstante, si se forma al profesorado y se le da material curricular para el aula, es verdad que tenderemos a lo que sería ideal en educación, cada alumno con su material idóneo para que él aprenda, además de interactuar con los demás estudiantes de su clase y así aprender de personas que opinan de distinta forma, con esto último se pueden desarrollar competencias como las de aprender a ser y aprender a vivir que son difíciles de integrar en el aula cuando el único fin es utilizar un ordenador o un tablet.

\section{Bibliografía}

AGUADED-GÓMEZ, J. I.; PÉREZ-RODRÍGUEZ, M. A.; MONESCILLO-PALOMO, M. (2010). "Hacia una integración curricular de las TIC en los centros educativos andaluces de Primaria y Secundaria”. En: Bordón no62 (4), p. 7-23.

AGUIAR, M.; CUESTA., H. (2009). "Importancia de trabajar las TIC en Educación Infantil a través de Métodos como las WebQuest". En: Revista de Medios y Educación no 34, p. 81-94.

CLARES, J.; GIL, J. (2008). "Recursos tecnológicos y metodologías de enseñanza en titulaciones del ámbito de las ciencias de la educación". En: Bordón nº 60 (3), p. 21-33.

CLIMENT GINE, G. (2002). "Des de l'esfera dels valors". En: Revista de Blanquerna $\mathrm{n}^{\circ} 7$, Barcelona.

COLÁS, P. (2002). "La investigación educativa en la (nueva) cultura científica de la sociedad del conocimiento. XXI. En: Revista de educación, no 4: p. 77-93. 
DONNELLY, D.; McGARR, O.; O’REILLY, J. (2011). “A framework for teachers' integration of ICT into their classroom practice". En: Computers \& Education, n" 57 [2], p. 1469-1483.

ECHEVARRÍA, J. (2000): "Conocimiento en el medio ambiente digital” En: Nueva Revista. (Madrid) nº70, p. 25-29.

EDMUNDS, R.; THORPE, M.; CONOLE, G. (2012). "Student attitudes towards and use of ICT in course study, work and social activity: a technology acceptance model approach" En: British journal of educational technology n43 (1), p. 71-84.

EISELE-DYRLI, K. (2011). “Mobile Goes Mainstream". En: District Administration, $\mathrm{n}^{\mathrm{o}} 47(2)$, p. 46-55.

ELLUL, J. (1980). The technological system (J. Neugroschel, Trans.). New York: Continuum.

EURYDICE (2011). Science education in Europe. EACEA Bruselas.

HAVU-NUUTINEN, S.; KEINONEN, T. (2010) "The changes in pupils' conceptions of human body based on science, technology and society based teaching". En: Journal of Baltic science education, $\mathrm{n}^{\circ} 9$ (3), p. 212-225.

INE. (2011). Estadística de la enseñanza universitaria en España: curso 2009-2010. Recuperado de de: http://www.ine.es/jaxi/menu.do?type=pcaxis\&path=/t13/ p405/a2009-2010\&file=pcaxis [Consulta: 22/12/2011].

JOSEPHSEN, J.; KRISTENSEN, A. (2006). "Simulation of laboratory assignments to support students' learning of introductory inorganic chemistry". En: Chemistry education research and practice, $\mathrm{n}^{0} 7$ [4], p. 266-279.

KELLER, T.; PEARSON, G. (2012) “A framework for k-12 science education: increasing op-portunities for student learning". En: Technology and engineering teacher, $\mathrm{n}^{\mathrm{0}} 1, \mathrm{p} .12-20$.

KOLB, L. (2011). "Adventures with cell phones". En: Educational Leadership, $\mathrm{n}^{\mathrm{o}} 68(5)$, p. 39-43.

KORTEMEYER, G. (2006). "An analysis of asynchronous online homework discussions in introductory physics courses". En: American journal of physics, nº74 [6], p. 526-536.

MARINO, M. T.; HAYES, M. T. (2012). "Promoting inclusive education, civic scientific literacy and global citizenship with videogames". En: Cultural studies of science education, DOI 10.1007/s11422-012-9429-8.

MARTÍN-VILLALBA, C.; URQUÍA, A.; DORMIDO, S. (2012). "Development of virtual-labs for education in chemical process control using Modelica". En: Computers and chemical engineering, n³9, p. 170-178.

MATTHEW, A. F. (2012) "Managing distraction and attention in diverse cohorts : 21 st century challenges to law student engagement". En: Queensland University of Technology Law and Justice Journal, n ${ }^{\circ} 12(1)$, p. 45-65.

MEI, L. (2009). "Bridging disciplinary boundaries". En: Education Canada, n49 (3), p.40-43.

MELHUISH, K.; FALLOON, G. (2010). "Looking to the future: M-learning with the iPad. Computers in New Zealand Schools: Learning, Leading, Technology, 22(3), 
1-16. Prensky, M. (2004) What can you learn from a cell phone? Almost anything! Retrieved from http://www.marcprensky.com/writing/, [Consulta 13-10-2012]. MEMBIELA, P. (1997). "Una revisión del movimiento educativo ciencia-tecnología-sociedad”. En: Enseñanza de las ciencias, nº15 (1), p. 51-57.

MÉNDEZ, D. (2012a). "Cambio motivacional realizado por las TIC en los alumnos de secundaria de Física". En: Miscelánea de Comillas, nº70 (136), p.199-224.

(2012b). "The experience of learning physics through the application of ICT". En: Energy education science and technology Part B. Social and educational studies, $\mathrm{n}^{\mathrm{o}} 4(1)$, p. 674-679.

(2012c). "La sociedad tecnológica actual y sus implicaciones en la educación científica". En: Sociedad y utopía, no 40 , p. 72-85.

(2013). "The influence of teaching methodologies in the learning of thermodynamics in secondary education". En: Journal of Baltic Science Education, $\mathrm{n}^{\circ} 12$ (1), p. 59-72.

(2014). "The effect of the computers in the learning of formulation with secondary students". En: Journal of science education, $\mathrm{n}^{\circ} 14$ (1).

MÉNDEZ, D.; GARCÍA-ALONSO, P. (2013). "Cambio comunicativo en las nuevas metodologías didácticas". En: Estudios sobre el mensaje periodístico, nº19, p. 299-308.

MÉNDEZ, D.; SLISKO, J. (2013). "Software Socrative and Smartphones as Tools For Implementation of Basic Processes of Active Physics Learning in Classroom: An Initial Feasibility Study With Prospective Teachers". En: European Journal of Physics Education, no 4 (2), p. 17-24.

ORLIK, Y. (2002). Chemistry: Active methods of teaching and learning. México: Iberoamérica Publ.

PEDERSEN, J. E.; TOTTEN, S. (2001) "Beliefs of science teachers toward the teaching of science/technological/social issues: are we addressing national standards?". En: Bulletin of science technology \& society, $\mathrm{n}^{\circ}$ 21: 376 . DOI: $10.1177 / 027046760102100507$.

PEDRETTI, E.; NAZIR, J. (2011) “Currents in STSE education: mapping a complex field, 40 years on". En: Science education, no 95(4), p. 601-626.

PROJECT TOMORROW (2010). Creating our future: Students speak up about their vision for 21 st century learning. Retrieved from http://www.tomorrow.org/ speakup/pdfs/SU09NationalFindingsStudents\&Parents.pdf [Consulta 13-102012]

SCHERER, R.; TIEMANN, R. (2012). "Factors of problem-solving competency in a virtual chemistry environment: the role of metacognitive knowledge about strategies". En Computers \& education, n59, p. 1199-1214.

SIGALÉS, C.; MOMINÓ, J. M. (2009). La integración de Internet en la educación escolar española: situación actual y perspectivas de futuro. Barcelona: Planeta.

SU, K. (2008). "The effects of a chemistry course with integrated information communication technologies on university students' learning and attitudes". En: International journal of science and mathematics education, $\mathrm{n}^{\circ} 6$ [2], p. 225-249. 
SWEENEY, A.; SEAL, S.; VAIDYANATHAN, P. (2003). "The promises and perils of nanos-cience and nanotechnology: exploring emerging social and ethical issues". En: Bulletin of science technology \& society, $n^{\circ} .23$, p. 236. DOI: 10.1177/0270467603256078.

VÁZQUEZ, Alberto (2011). Plan-Do-Check-Act en una experiencia TIC en el aula: desde la idea a la evaluación. En: EDUTEC, Revista Electrónica de Tecnología Educativa 36, junio de 2011. http://edutec.rediris.es/revelec2/revelec36 [Consulta 26-10-2011]

WILDER, A.; BRINKERHOFF, J. (2007). "Supporting representational competence in high school biology with computer-based biomolecular visualizations". En: Journal of computers in mathematics and science teaching, $\mathrm{n}^{\circ} 26$ [1], p.5-26.

YASUDA, H. (2010). "A risk management system to oppose cyber bullying in high school: warn-ing system with leaflets and emergency staffs". En: Informatica, no34, p. 255-259.

ZACHARIA, Z.; OLYMPIOU, G. (2011). "Physical versus virtual manipulative experimentation in physics learning". En: Learning and instruction, $\mathrm{n}^{\circ} 21$, p.317331.

\section{Los autores}

David Méndez Coca. Licenciado en Ciencias Físicas en la UCM (2002) y doctor en Educación en la UCM (2011). Premio extraordinario de doctorado. Profesor de secundaria y bachillerato durante diez años. Autor de más de diez artículos publicados en revistas españolas y extranjeras, más de quince comunicaciones en congresos en México, Estados Unidos, República Checa, España, etc. Profesor en el Centro Universitario Villanueva desde el 2011.

Miriam Méndez Coca. Nacida el 2/2/1975 en Madrid (ESPAÑA). Licenciada en Matemáticas en Universidad Complutense de Madrid y doctora en Pedagogía en Universidad Pontificia de Salamanca con la tesis "Enseñanza-aprendizaje de las matemáticas en la E.S.O." defendida el 2013 obteniendo una calificación de Cum Laude. Trabajé como analista de programación para Unión Fenosa y BBVA 2000 2009. Máster en Ingeniería de Software 2002-2004. Profesora de secundaria y bachillerato desde 2010 en diferentes centros públicos, privados y concertados. Profesora del centro universitario de Villanueva (UCM) desde el 2013. Título de inglés de la Escuela Oficial de Idiomas. 